\title{
Gestão de resíduos da construção civil: análise bibliométrica entre 2010 e 2020
}

\author{
Civil construction waste management: bibliometric analysis between 2010 and 2020
}

Gestión de residuos de la construcción: análisis bibliométrico entre 2010 y 2020

\section{Resumo}

A indústria da construção civil contribui para a degradação ambiental devido aos impactos causados ao ambiente e ao volume de resíduos descartados sem tratamento adequado. Esse cenário influencia pesquisadores por meio de estudos ligados à temática Gestão de Resíduos da Construção Civil (GRCC) a encontrar formas de reduzir estes impactos. Baseado nisso, este estudo buscou identificar as tendências de pesquisa sobre a temática GRCC e apresentar a análise da evolução das publicações, por meio do mapeamento dos países, autores e coocorrência das palavras-chave. A metodologia utilizada foi o estudo bibliométrico a partir de produções científicas da base de dados Scopus, considerando o período entre 2010 e 2020. Os aspectos analisados foram ano de publicação, publicações por países, publicações por periódicos, e as relações de citação, autoria e coocorrência das palavras-chave por meio da análise dos mapas de rede. Os resultados apontaram que há uma tendência de crescimento na quantidade de artigos publicados a partir de 2016, sendo a China o país com maior destaque no volume de publicações, e apresentando autores de referência, contribuindo ainda com uma visão abrangente sobre os caminhos de pesquisas futuras sobre temática GRCC.

Palavras-chave: Bibliometria; RCC; Resíduo de construção; Resíduo de demolição.

\begin{abstract}
The civil construction contributes to environmental degradation due to the impacts caused to the environment and to the volume of waste disposed of without adequate treatment. This scenario influences researchers through studies linked to the thematic of Civil Construction Waste Management (CCWM) to find ways to reduce these impacts. Based on this, this study sought to identify research trends on the CCWM thematic and to present an analysis of the evolution of publications, through the mapping of countries, authors and co-occurrence of keywords. The methodology used was the bibliometric study based on scientific productions from the Scopus database, considering the period between 2010 and 2020. The aspects analyzed were year of publication, publications by countries, publications by journals, and the relations of citation, authorship and co-occurrence of the keywords through the analysis of network maps. The results pointed that there is an growth trend in the number of articles published from 2016, with China being the country with the highest volume of publications, and featuring reference authors, also contributing with a comprehensive view of future research paths on CCWM thematic.
\end{abstract}

Keywords: Bibliometric; CDW; Construction waste; Demolition waste.

\section{Resumen}

La industria de la construcción civil contribuye a la degradación del medio ambiente debido a los impactos causados al entorno y al volumen de residuos eliminados sin un tratamiento adecuado. Este escenario influye en los investigadores a través de estudios vinculados al tema Gestión de Residuos de la Construcción Civil (GCR) para encontrar formas de reducir estos impactos. A partir de ahí, este estudio pretendía identificar las tendencias de la investigación sobre el tema de la GRCC y presentar el análisis de la evolución de las publicaciones mediante el mapeo de los países, los autores y la co-ocurrencia de palabras clave. La metodología utilizada ha sido un estudio bibliométrico basado en las producciones 
científicas de la base de datos Scopus, considerando el periodo comprendido entre 2010 y 2020. Los aspectos analizados fueron el año de publicación, las publicaciones por países, las publicaciones por revistas y las relaciones de citación, autoría y co-ocurrencia de las palabras clave a través del análisis de mapas de red. Los resultados señalaron que existe una tendencia de crecimiento en la cantidad de artículos publicados a partir de 2016, siendo China el país con mayor protagonismo en el volumen de publicaciones, y presentando autores de referencia, contribuyendo aún con una visión integral sobre los caminos de la investigación futura en el tema de GRCC.

Palabras clave: Bibliometría; RCC; Residuos de la construcción; Residuos de la demolición.

\section{Introdução}

O sistema de construção atual tem sido considerado como um dos principais contribuintes para a degradação ambiental, sendo ainda responsável pelo grande consumo de energia e de recursos naturais que gera níveis inaceitáveis de resíduos sólidos, desperdiçados quando despejados em aterros controlados ou não controlados (Llatas, 2011; Cheng, et al., 2013; Yuan, et al., 2012).

Apesar de reconhecer a contribuição significativa que a indústria da construção civil tem para o ambiente construído, esta atividade também é responsável por impactos causados ao ambiente natural devido ao volume de Resíduos da Construção Civil (RCC) descartado sem tratamento adequado (Lu, et al., 2011; Yuan, et al., 2012; Barbudo, et al., 2020).

A construção civil é responsável por $40 \%$ do total de resíduos gerados no mundo. Devido às lacunas no dimensionamento e no conhecimento de sua quantidade real, uma porcentagem baixa é recuperada, a maior parte é destinada para os aterros sanitários onde ocupam um volume que excede a dos resíduos domésticos (Ya’Cob, et al., 2013; Solís-guzmán, et al., 2009; Zega \& Di Maio, 2011; Llatas, 2011; Ortiz, 2010).

Essa quantidade de resíduos tem suscitado, desde a década de 1980, esforços contínuos de pesquisadores dedicados a encontrar formas de reduzir os impactos adversos associados à construção, por meio de uma ampla gama de estudos ligados à temática Gestão de Resíduos da Construção Civil (GRCC), com a utilização de paradigmas e métodos de pesquisa diversos, assim como diferentes perspectivas e conteúdos que incluem desde a redução da geração, passando pela reciclagem até a eliminação, ou seja, a disposição final ambientalmente adequada (Yuan, et al., 2012; Chen, et al., 2018).

Para Yuan e Shen (2011), o aumento no volume de publicações nesta temática em um curto período de tempo apresenta desafios aos pesquisadores, por não possibilitar uma visão geral das pesquisas já desenvolvidas, tornando assim, segundo Chen, et al. (2018), importante analisar os últimos avanços e as tendências emergentes, com o propósito de estimular estudos futuros e práticas inovadoras.

Nessa vertente, a análise bibliométrica apresenta-se como um método para conhecer e compreender o domínio de conhecimento de um assunto específico, permitindo que o desenvolvimento geral de publicações possa ser analisado de forma quantitativa e sistemática, utilizando-se de métodos matemáticos e estatísticos para investigar as características, estrutura, tópicos importantes e tendências de pesquisa (Wu, et al., 2019; Chen, et al., 2018).

Assim surge o questionamento: Quais as temáticas e tendências de pesquisa relacionadas à GRCC no panorama mundial?

Como uma tentativa de reflexão sobre os RCC e compreensão do foco dos pesquisadores expresso por meio de artigos acadêmicos, este estudo busca identificar as tendências de pesquisa sobre a temática GRCC e apresentar a análise da evolução das publicações, por meio do mapeamento dos principais países, autores e coocorrência das principais palavras-chave.

\section{Metodologia}

Esta pesquisa se caracteriza por uma abordagem quanti-qualitativa com o uso de técnicas bibliométricas. Com base em Prodanov e Freitas (2013), é classificada quanto à sua natureza como pesquisa básica, com a intenção de gerar conhecimento para o progresso da ciência sem aplicação prática. No que se refere aos seus objetivos é exploratória-descritiva, pois pretende 
proporcionar a compreensão das tendências de pesquisa sobre a GRCC no panorama mundial. Quanto aos procedimentos técnicos, é qualificada como bibliográfica por ser elaborada a partir de fontes publicadas (Da Silva \& Menezes, 2005).

Em relação ao desenvolvimento temporal caracteriza-se como longitudinal, devido às análises das produções científicas terem considerado o período de 2010 a 2020. Como etapa precedente à coleta de dados, definiu-se a Scopus como base de dados a ser consultada, por ser o maior banco de dados atualmente, permitindo melhores resultados.

Na primeira etapa, definiu-se os termos para a busca, sendo estes: "Construction Wast*" OR "Demolition Wast*" AND "Management", utilizando a pesquisa de forma simples no campo "article title, abstract e keywords". O filtro para refinamento da pesquisa foi o recorte temporal, considerando também apenas artigos na língua inglesa.

A busca resultou em 758 artigos, os quais foram tratados estatisticamente com base no ano de publicação, publicação por países e publicação por periódicos. Posteriormente, iniciou-se a segunda etapa com a seleção dos artigos, por meio da leitura dos títulos, resumos e palavras-chave. Os artigos que não possuíam como temática "resíduos da construção civil", foram excluídos manualmente da amostra. Dos 758 artigos publicados, restaram 655 após o processo de exclusão.

Os artigos que se encaixavam dentro dos critérios estabelecidos para a pesquisa foram selecionados e exportados da Scopus em um arquivo CSV, por ser este o formato suportado pelo software VOSViewer@ que é uma ferramenta utilizada como apoio ao estudo bibliométrico na construção de mapas de rede.

A terceira etapa compreendeu a análise das redes bibliométricas geradas no VOSviewer e demonstraram as relações de citação, autoria e coocorrência das palavras-chave, mineradas da literatura científica sobre GRCC.

Os resultados estão apresentados de forma quantitativa, por meio de gráficos que demonstram os principais periódicos, a evolução das publicações ao longo do período estabelecido, os principais países, e os mapas de rede e sobreposição.

\section{Resultados e Discussão}

Os resultados encontrados referem-se à análise dos artigos no tocante à GRCC, encontrados na base de dados Scopus, e sistematizados com o auxílio do software VOSViewer@. Ressalta-se que os resíduos de demolição são aqui contemplados pelo termo "Resíduos da Construção Civil - RCC".

$\mathrm{Na}$ quantidade de artigos publicados por ano (Figura 1) se verificou uma tendência de crescimento, especialmente a partir do ano de 2016. Esta tendência persistiu até o final do período analisado indicando que é uma temática de interesse de pesquisadores.

Figura 1 - Panorama das publicações sobre GRCC (2010-2020)1.

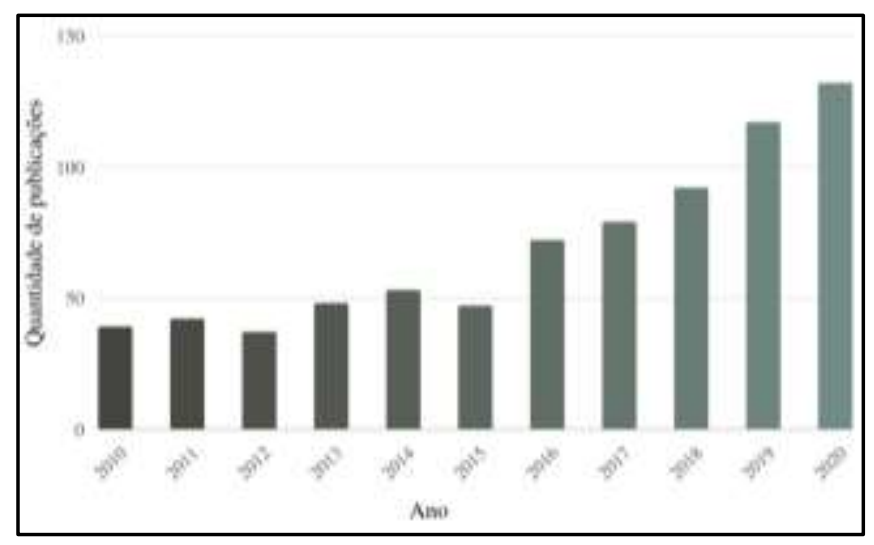

Fonte: Scopus (2020) adaptado por Autoras (2020). 
Além do aumento de publicações ao longo dos anos pesquisados, a preocupação com a GRCC é uma temática de interesse mundial, tendo como registro 74 países (Figura 2). Dentre os países com as maiores frequências de publicações encontram-se países como a China (26\%), seguido da Espanha (9\%), Reino Unido, Austrália e Brasil (7\%), dentre outros.

Destaca-se que o aumento no volume de publicações a partir de 2016 pode ter relação com o interesse de acadêmicos da China, especialmente em Hong Kong que tem demonstrado amplo interesse em investigar as práticas, desafios e estratégias da GRCC, conforme encontrado também nos estudos de Chen, et al. (2018).

Figura 2 - Publicação sobre GRCC por países (2010-2020)2.

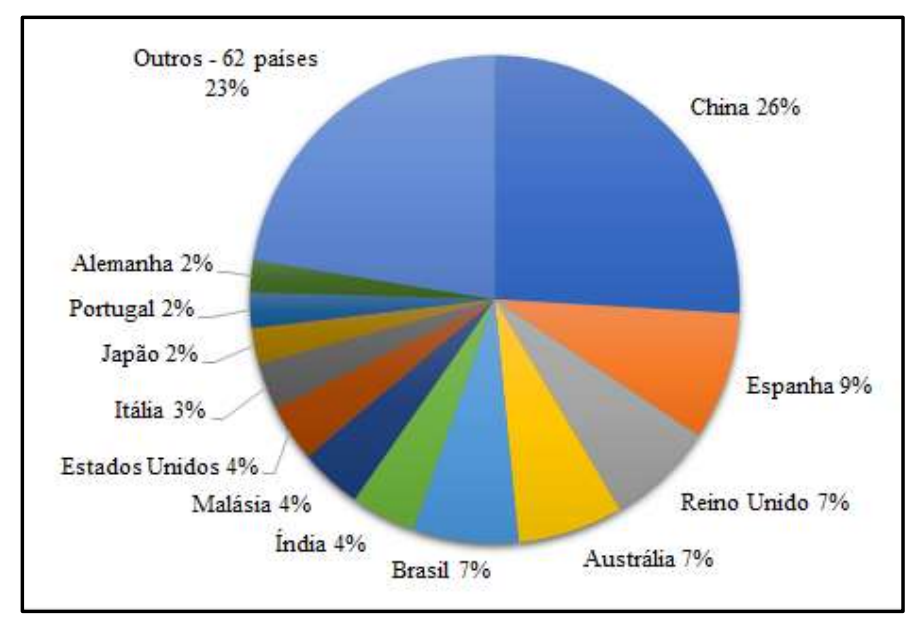

Fonte: Scopus (2020) adaptado por Autoras (2020).

A China, incluindo as publicações de Hong Kong (território da China), foi o país com maior número de publicações com 33\% (Figura 2). Tem se destacado, desde 2006, como um dos países mais promissores em termos de desempenho científico, assumindo a posição de liderança mundial em alguns campos especiais (Zhou \& Leydesdorff, 2006; Huang, et al., 2010; Mervis, 2010).

O desempenho científico da China nas pesquisas com a temática voltada para a GRCC está relacionado com a enorme quantidade de RCC devido ao crescimento da população, em particular a urbana, e o crescimento do Produto Interno Bruto (PIB) após a década de 1980 (Zega \& Di Maio, 2011). Estudos realizados por Wang, et al. (2018) relatam que a quantidade de RCC gerada na China está entre 2 a 3 bilhões t/ano.

Na sequência, os achados da pesquisa referentes à Espanha (9\%) e ao Reino Unido (7\%) demonstram que países desenvolvidos se destacam com maior número de publicações, isto pode estar relacionado a cooperação entre instituições de pesquisa (Hou, et al., 2015). Especificamente no Reino Unido, a indústria da construção civil é responsável pela geração de cerca de $32 \%$ do total de resíduos depositados em aterros e $25 \%$ de toda a matéria-prima (Lu, et al., 2017).

A Austrália e o Brasil também contribuíram com 7\% das publicações. Na Austrália, os resíduos gerados constituem cerca de $44 \%$ da quantidade total de rejeitos ao ano (Park \& Tucker, 2017). Enquanto no Brasil, a produção de RCC representa em torno de 50\% a 70\% do total de resíduos sólidos urbanos, o que desperta a atenção da academia, gestores públicos e da sociedade acerca dos impactos ambientais oriundos do descarte irregular (Brasileiro \& Matos, 2015; ABRELPE, 2019).

A China, o Brasil e a Índia são países considerados de economia emergente que estão em processo de expansão urbana e construção de infraestrutura, mas se distinguem em relação a produção acadêmica relacionada ao RCC. Ghafourian (2016)

${ }^{2}$ Foram especificados os países com 20 ou mais publicações, os demais (62 países; 209 publicações; média 3,37 publicações por país) estão agrupados na categoria "Outros". Dados: 74 países; um total de 655 publicações. 
afirma que isso demonstra a importância dada à GRCC nestes países em comparação com outros que também estão em desenvolvimento.

A introdução de legislações de gestão de resíduos também contribui para o aumento do número das publicações. Ghafourian (2016) constatou que a falta de publicações sobre GRCC em países em desenvolvimento pode estar relacionada a falta de regulamentações ambientais mais rígidas e de conscientização adequada, enquanto Chen, et al. (2018) verificou o aumento significativo de pesquisas a partir de 2006 com o Esquema de Cobrança de Eliminação de Resíduos promulgado em Hong Kong, a publicação da Estratégia de Resíduos para a Inglaterra no ano de 2007, a diretiva-quadro de resíduos que foi normatizada pela União Europeia em 2008 e no Brasil a instituição da Política Nacional de Resíduos Sólidos, no intuito de regulamentar sobre o gerenciamento adequado dos resíduos.

No tocante aos periódicos, foram identificados entre os 758 artigos analisados um total de 160 periódicos (Figura 3).

Figura 3 - Periódicos com maior número de publicações sobre GRCC (2010-2020).

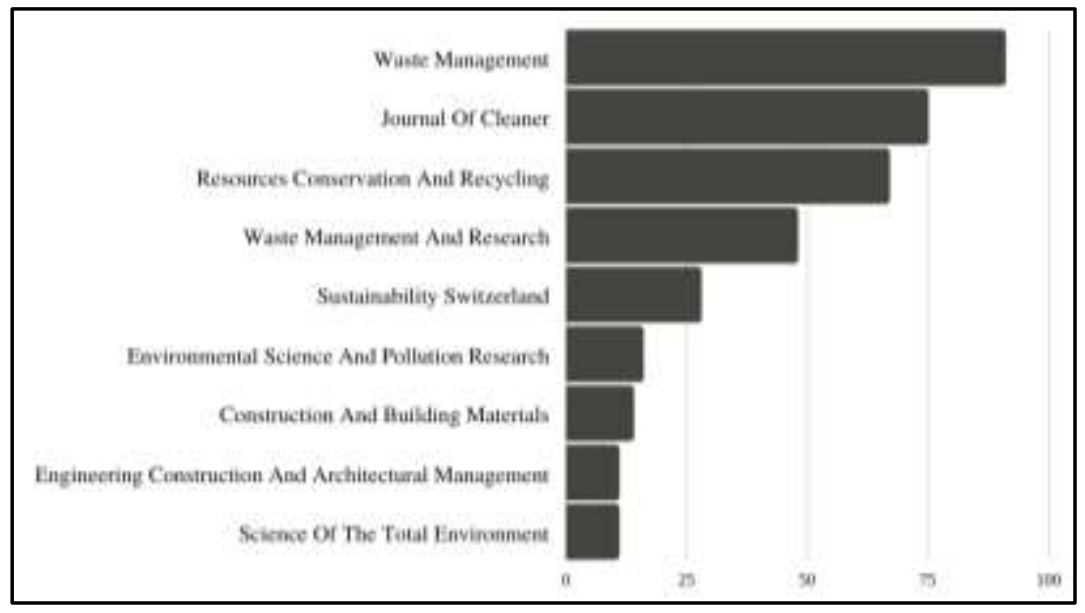

Fonte: Scopus (2020), adaptado por Autoras (2020).

Dentre os periódicos que mais contribuíram destaca-se o Waste Management com 91 publicações, esse valor corresponde a $12 \%$ do total de produções analisadas. Na sequência estão os periódicos Journal of Cleaner Production e Resources Conservation and Recycling com 10\% e 9\% do total, respectivamente. As áreas de publicação têm em seu escopo a das ciências ambientais.

Estes resultados se assemelham aos apresentados por Chen, et al. (2018) em que os periódicos Resources Conservation and Recycling, Waste Management, Journal of Cleaner Production se destacam com as publicações mais conceituadas e prestigiadas, sendo que a Waste Management e Resources Conservation and Recycling apresentam bom desempenho não só no número de artigos publicados, como também na qualidade da pesquisa na temática GRCC.

Já os resultados de Wu, et al. (2019) apresentam quatro periódicos com contribuições significativas para a disseminação de pesquisas de RCC, sendo estes, respectivamente: Construction and Building Materials, Waste Management, Journal of Cleaner Production e Resources Conservation and Recycling.

\subsection{Análise de redes}

A partir da análise de redes de coautoria foi possível identificar os pesquisadores, instituições de pesquisa ou países que estão relacionados, com base na quantidade de estudos que realizam e publicam em conjunto (Van Eck \& Waltman, 2014).

Para a criação dos mapas baseados nas coautorias, usou no software a opção countries relacionadas aos autores além do método de full counting. Do total de 74 países, 38 foram selecionados pelo VOSViewer@ com base no limite de corte a partir de 
oito publicações por país, além disso, foi realizado um filtro manual excluindo os países duplicados. As redes de coautoria dos artigos com os países indicaram 34 nós organizados em oito clusters (Figura 4).

Figura 4 - Mapa de rede de autores e países. Quanto maior o tamanho das esferas, mais representativo ${ }^{3}$.

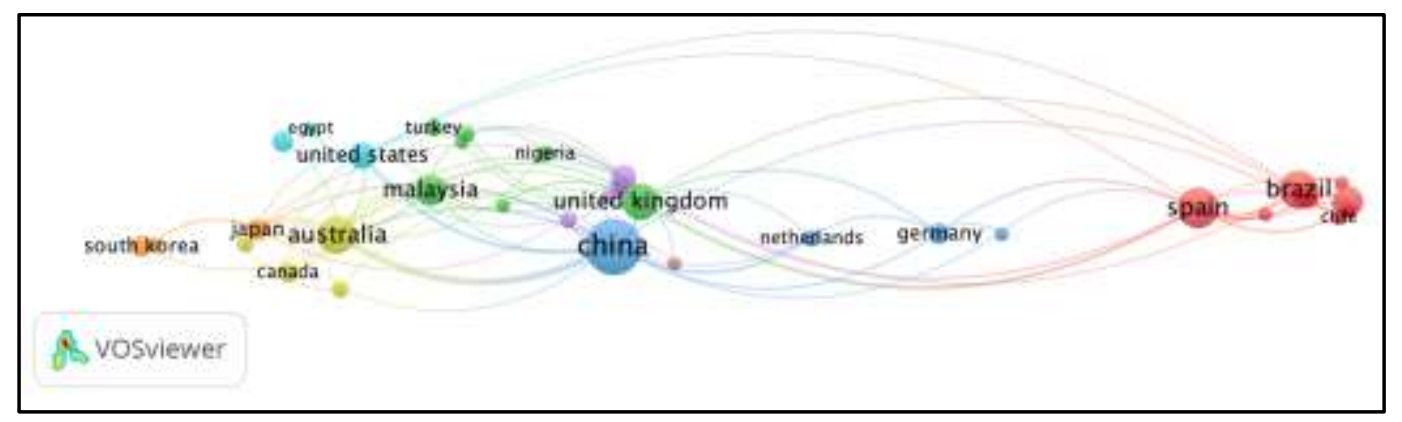

Fonte: Scopus; Vosviewer (2020) elaborado pelas Autoras (2020).

Por meio da análise dos clusters, identificou-se os países com maior frequência de estudos nesta temática e a interrelação entre os que têm realizado pesquisas de forma colaborativa. O resultado mostra a relação de países desenvolvidos com países em desenvolvimento, sobretudo no que se refere à força do link. Sobre esse dado, pode-se inferir que a cooperação entre instituições de pesquisa facilita a comunicação dos pesquisadores e a articulação acadêmica.

A sobreposição da rede de países e coautorias com relação à média temporal das publicações (Figura 5), indica que no ano de 2015 se destacaram Canadá, Alemanha, Países Baixos, Bélgica e Argentina. Já os países com publicações mais recentes, anos de 2017 e 2018, tem-se o Vietnã, Egito, Austrália e Chile (Figura 5).

Figura 5 - Mapa de sobreposição de autores e países ${ }^{4}$.

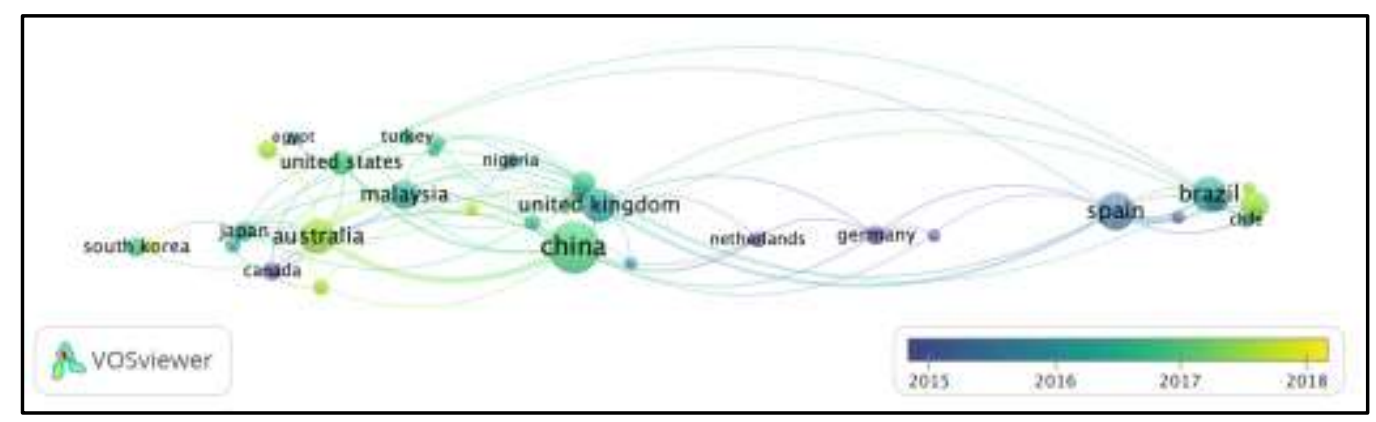

Fonte: Scopus; Vosviewer (2020) elaborado pelas Autoras (2020).

Outra análise realizada foi a de coocorrência de palavras-chave que é estabelecida por meio do número de artigos indexados na base de documentos, que ocorrem conjuntamente tanto no título quanto no resumo ou palavras-chave (Van Eck \& Waltman, 2014). As palavras-chave apontam o resumo do conteúdo da pesquisa e o desenvolvimento dos temas ao longo do tempo (Chen, et al., 2018). Para a criação dos mapas baseados nas coocorrências das palavras-chave, elegeu-se no VOSViewer@ a opção all keywords além do método de full counting que atribui o mesmo peso para cada link em coocorrência. Do total de

3 As cores identificam a separação por clusters. Cluster 1, de cor vermelha, oito países - Brasil, Espanha, Chile, Argentina, Portugal, Índia, Colômbia e França; Cluster 2, de cor verde, sete países - Reino Unido, Malásia, Turquia, Nigéria, Paquistão, Arábia Saudita e Taiwan; Cluster 3, de cor azul escuro, quatro países - China, Bélgica, Alemanha e Países Baixos; Cluster 4, de cor amarela, quatro países - Austrália, Áustria, Canadá e Tailândia; Cluster 5, de cor roxa, quatro países - Dinamarca, Finlândia, Itália e Romênia; Cluster 6, de cor azul claro, três países Egito, Irã e Estados Unidos; e Cluster 7, de cor laranja, três países - Japão, Coreia do Sul e Vietnã; Cluster 8, de cor marrom, um país Singapura.

${ }^{4} \mathrm{O}$ gradiente de cores identifica a escala temporal, sendo que as cores mais claras representam as tendências mais recentes. 
1670, 45 foram selecionadas pelo software com base no limite de corte de sete ocorrências por palavra-chave. Foi feita a retirada manual de construction and demolition waste $(c d w)$ por esta se apresentar de forma duplicada, restando assim 44 termos.

Nas redes de coocorrência de palavras-chave (Figura 6), foram contabilizados 283 nós organizados em seis clusters. Essa representação determina a temática central das produções, na qual as palavras-chave se apresentam com maior frequência. $\mathrm{Na}$ análise dos clusters foram identificadas as áreas de estudo e os prováveis conjuntos de temas mais comuns e relevantes sobre GRCC.

Figura 6 - Redes de co-ocorrências de palavras-chave. ${ }^{5}$

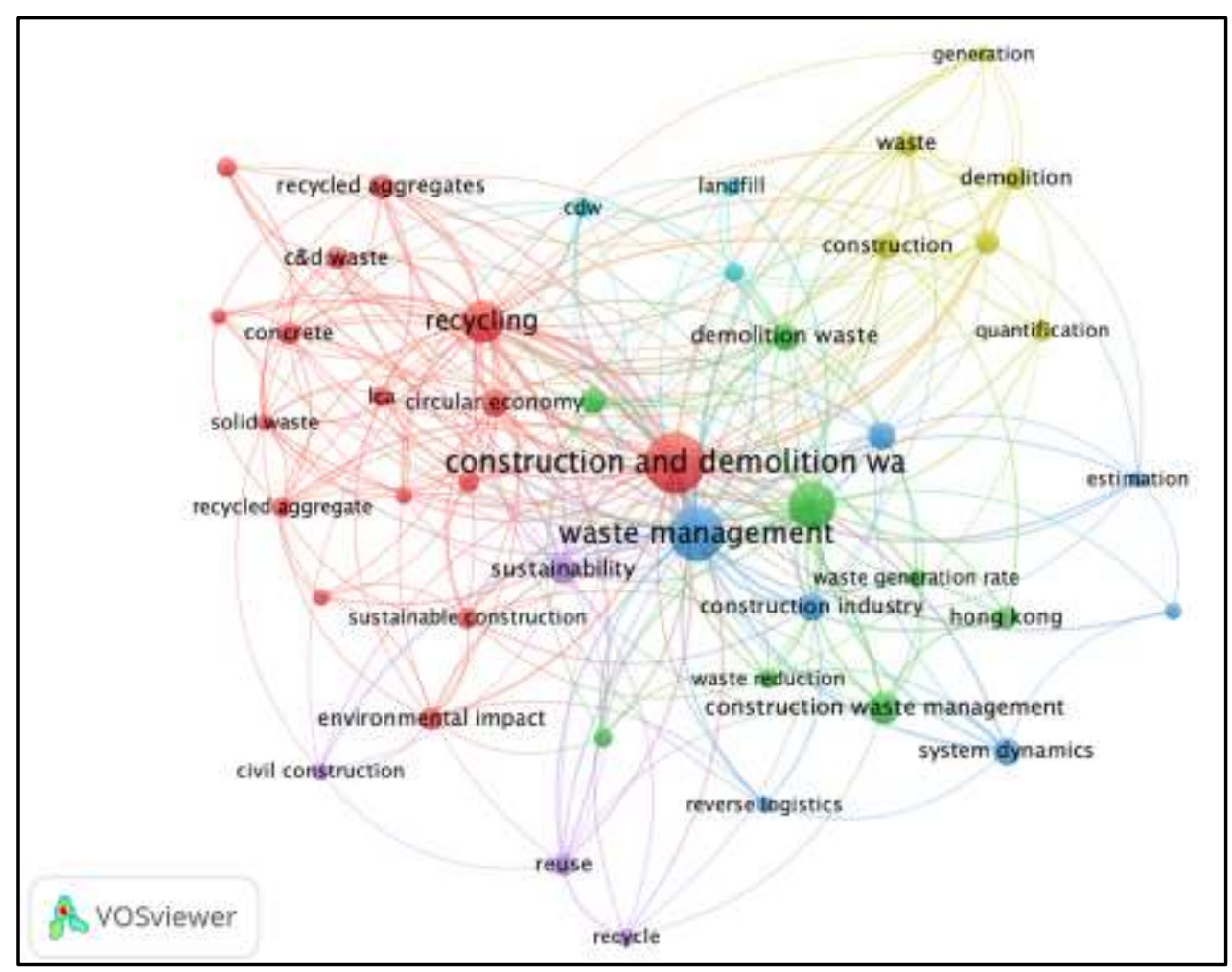

Fonte: Scopus; Vosviewer (2020) elaborado pelas Autoras (2020).

O primeiro cluster, mais numeroso, é caracterizado pelas pesquisas sobre "construction and demolition waste", "recycling", "circular economy", "recycled aggregates", "sustainable construction", e "environmental impact", dentre outros, com um menor número de coocorrências (Figura 6). Esse cluster apresenta-se com destaque por representar um conjunto maior de palavras-chave, o que pode indicar considerando a abordagem apresentada pelos autores uma busca de soluções em termos de gestão para os RCC. De acordo com Chen, et al. (2018), a palavra-chave "reciclagem” aparece com frequência por atrair grande parte dos esforços de pesquisa.

No segundo cluster estão as palavras-chave associadas com maior frequência de ocorrência, e referem-se a pesquisas sobre "construction waste", "demolition waste", "life cycle assessment", "waste generation rate", "hong kong", entre outros

\footnotetext{
${ }^{5}$ Cluster 1 , de cor vermelha, 16 termos - construction and demolition waste, circular economy, recycling, recycled aggregates, c\&d waste, concrete, solid waste, recycled aggregate, sustainable construction, environment impact, ICA, dentre outros; Cluster 2, de cor verde, 8 termos - construction waste, demolition waste, waste generation rate, hong kong, waste reduction, construction waste management, life cycle assessment, dentre outros; Cluster 3, de cor azul, 7 termos - waste management, reverse logistics, sustainable development, estimation, system dynamics, dentre outros; Cluster 4, de cor amarela, 6 termos - construction, demolition, generation, quantification; Cluster 5, de cor lilás, 4 termos - sustainability, recycle, civil construction e reuse; Cluster 6, de cor azul claro, 3 termos - landfill, cdw e construction management.
} 
(Figura 6). Esse cluster associa um conjunto de temas ligados à uma preocupação com os resíduos desde a sua origem até o seu descarte, o que pode indicar uma tendência mais clara de inclusão de questões ambientais.

Dentre os termos apresentados, destaca-se a ocorrência do termo Análise do Ciclo de Vida (life cycle assessment), considerado como tendência de pesquisa, principalmente pela sua atualidade no segmento da construção civil. Este fato pode ser observado por meio da revisão de artigos relacionados à Análise do Ciclo de Vida na GRCC realizada por Bovea e Powel (2016), quando demonstra que 66,3\% do total de artigos são realizados e publicados em países europeus como Espanha, Itália, Portugal e Suécia, 15\% são dos Estados Unidos e 10\% são da Ásia, predominantemente por pesquisadores da China. Laurent, et al. (2014) também demonstram esta preocupação, quando sugerem que são necessárias mais pesquisas com foco nos estudos de avaliação do Ciclo de Vida da GRCC.

A coocorrência da palavra "hong kong"como mais frequente neste cluster, indica que o território é foco de pesquisas por apresentar uma quantidade considerável de geração de resíduos da construção todos os anos, e que essa tendência de geração resultará na utilização de todos os aterros públicos e privados do território em alguns anos, o que tem atraído o interesse de pesquisadores (Chen, et al., 2018). Além das pesquisas, o território busca formular uma política de gestão de RCC, com base em princípios como o do 3R's (Redução, Reutilização e Reciclagem) e o do poluidor-pagador (Poon, et al., 2013; Ann, et al., 2013; Hossain, et al., 2017; Chen, et al., 2018).

No terceiro cluster encontram-se as pesquisas de assuntos ligados a "waste management", "reverse logistics", "sustainable development", e "system dynamics", dentre outros (Figura 6). Com base nesses clusters, atribui-se a preocupação dos autores com a associação com outras temáticas de pesquisa da área.

O quarto cluster é composto por "construction", "demolition", "generation", "quantification", dentre outros (Figura 6) e apresenta um conjunto de temas de pesquisas relacionadas ao próprio processo de monitoramento da GRCC.

No quinto cluster estão agrupados temas como, "sustainability", "recycle", "civil construction" e "reuse" (Figura 6). Por favorecer a sustentabilidade, a demanda por materiais reutilizáveis e recicláveis de alta qualidade tem sido crescente, entretanto depende de uma cadeia de produção definida, estruturada e com operadores logísticos eficientes. Estas temáticas apresentadas neste cluster demonstram a preocupação com relação à escassez de recursos naturais, impulsando assim a indústria da construção civil por colaborações efetivas e abrangentes entre cientistas, formuladores de políticas, ministros do governo e empresas, na busca por melhores alternativas, conceitos e soluções técnicas que possam garantir a sustentabilidade ambiental (Marques, et al., 2020; Ghaffar, et al., 2020).

O último cluster é composto por temas sobre "landfill", "construction management", "cdw" (Figura 6). No que diz respeito à GRCC, sugere-se que esta temática tem recebido a atenção da academia, devido a estimativa de 35\% das quantidades de RCC do mundo serem destinadas de forma indevida para áreas de aterros sem nenhum tratamento, causando sérios impactos (Córdoba, 2014; Bovea \& Powell, 2016; Hossain, et al., 2017; Menegaki \& Damigos, 2018). Além disso, o grande volume de RCC diminui a vida útil dos aterros planejados comprometendo-os para o recebimento de outros tipos de resíduos.

Para finalizar a caracterização das publicações sobre GRCC, destaca-se os termos em que a média de ocorrências aparece como mais atuais. Considerar esses termos com esse critério, ajuda a compreender o agrupamento, com base na relevância e na tendência de pesquisas futuras.

Os temas "circular economy", "waste generation rate" e "waste recycling" foram frequentes nos trabalhos mais recentes (Figura 7), embora este último seja um tema que predominou desde o início do período analisado, ainda continua no foco dos estudos e se apresenta como atual, indicando que as pesquisas ainda não exauriram as possibilidades de reciclagem para os resíduos da construção.

Considerando que o conceito de Economia Circular (circular economy) está ganhando incentivo global tanto na política, como nos negócios e na academia, o conhecimento e as ferramentas para colocá-lo em prática ainda precisam ser desenvolvidos, 
especialmente na indústria da construção civil, na qual as práticas inovadoras são difundidas muito lentamente e o foco tem sido em questões como uso de energia e eficiência energética (Bocken, et al., 2017; Lacy \& Rutqvist, 2015).

Figura 7 - Redes de coocorrências de palavras-chave ${ }^{6}$.

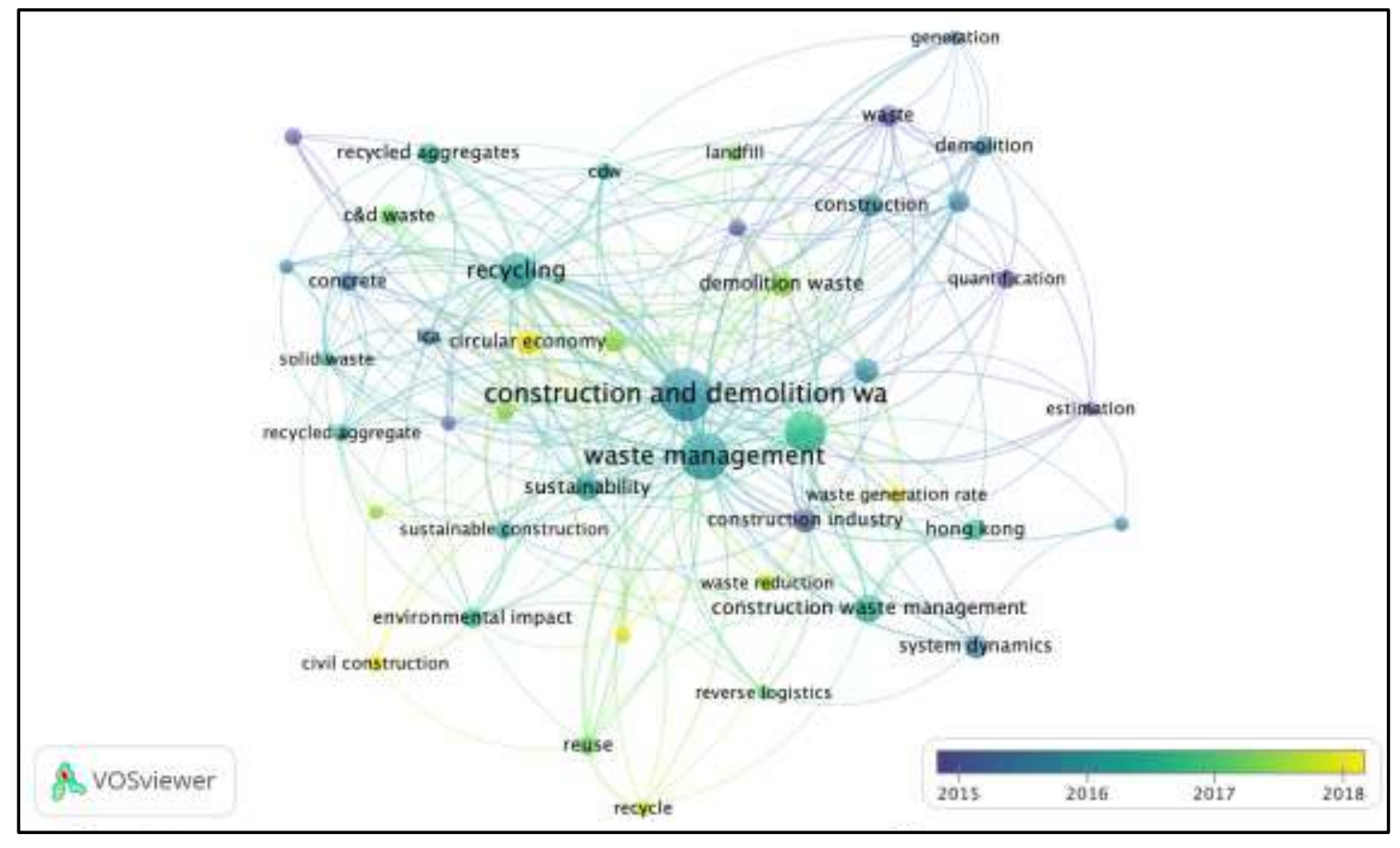

Fonte: Scopus; Vosviewer (2020) elaborado pelas Autoras (2020).

A representação das principais palavras-chave de 2015 a 2018 (Figura 7) demonstra que o estágio inicial da temática GRCC foi: waste, quantification, construction industry, estimation, dentre outros. A partir de 2016 os temas mudaram para construction and demolition, waste management, recycling, construction waste management, sustainability, dentre outros. As publicações de 2017 focaram nos temas como: reuse, demolition waste, landfill, c\&d waste, waste reduction, reverse logistics, dentre outros. O último ano representado, 2018, os temas mais recorrentes foram: circular economy, civil construction, waste generation rate e recycle.

Com base na análise de coocorrência das palavras-chave pode se inferir a heterogeneidade que as pesquisas apresentam, não sendo possível dizer com precisão todos os temas consensuais a temática GRCC.

Esta polissemia adquirida ao longo dos anos demonstra as abordagens diversas que os pesquisadores atribuíram à temática. Algumas dessas abordagens evoluíram e deram origem a pesquisas que vão além da compreensão da geração e quantificação dos RCC, e mesmo sendo etapas do processo de gestão de resíduos parecem consolidadas enquanto temas de pesquisas (cores lilás e verde escuro (Figura 7). Destaca-se que em comparação com os achados da pesquisa de Chen, et al. (2018), tem-se relação semelhante, em que os temas passam por uma alteração de conceitos básicos de GRCC para a análise de desafios (internos e externos) com foco na estratégia organizacional e práticas de gestão inovadoras.

No que se refere aos autores, o software VOSViewer@ identifica a quantidade de artigos publicados por cada autor, o número de citações e as ligações entre cada um. Para a construção dos mapas, com base nos autores e na coautoria, definiu-se o limite de três documentos publicados e no mínimo cinco citações por autor, totalizando assim a amostra com 124 autores. Foi realizado um filtro manual excluindo os autores duplicados da lista.

\footnotetext{
${ }^{6} \mathrm{O}$ gradiente de cores identifica a escala temporal, com as cores mais claras representando as tendências mais recentes.
} 
O autor com o maior número de publicações na temática de pesquisa foi Lu, W., entretanto, mesmo possuindo a maior quantidade de artigos, ele não é o autor com o maior número de citações, sendo este Yuan, H., autor referência sobre a temática. Uma das suas obras é Trend of the research on construction and demolition waste management, em tradução livre: A tendência de pesquisa sobre gestão de resíduo de construção e demolição, que foi a primeira revisão sistemática sobre o assunto, que analisou a tendência de pesquisa no período de 2000 a 2009 (Yuan \& Shen; 2011).

Embora sejam artigos metodologicamente diferentes, inclusive na estratégia de busca, têm-se em termos comparativos uma relação de continuidade no que tange às tendências de pesquisa apresentadas neste trabalho com as de Yuan e Shen (2011), como por exemplo: quantificação, taxa de geração, reciclagem e gestão sustentável dos resíduos da construção civil. Acrescentase aos achados desta pesquisa temas como logística reversa e economia circular, demonstrando um avanço nas tendências que não foi identificado por Yuan e Shen (2011). Outro dado apontado ainda por estes autores, corresponde aos países que mais publicaram sobre a temática, tendo como destaque Hong Kong (China), Austrália e EUA dentre os países desenvolvidos, com uma projeção no aumento de publicações da China e Malásia, em que o setor da construção civil é a principal atividade econômica, o que se se assemelha aos resultados encontrados nesta pesquisa.

O mapa de sobreposição da rede de autores e coautores relaciona os autores aos anos de maior destaque em suas publicações. Entre os autores classificados em uma escala temporal (Figura 8), os pesquisadores chineses se destacam tanto entre as publicações mais antigas (Yuan, Hao, Tam, Le, Shen), quanto as mais recentes (Liu, Bao, Chi, Gong).

Figura 8 - Mapa de sobreposição de autor e coautoria ${ }^{7}$.

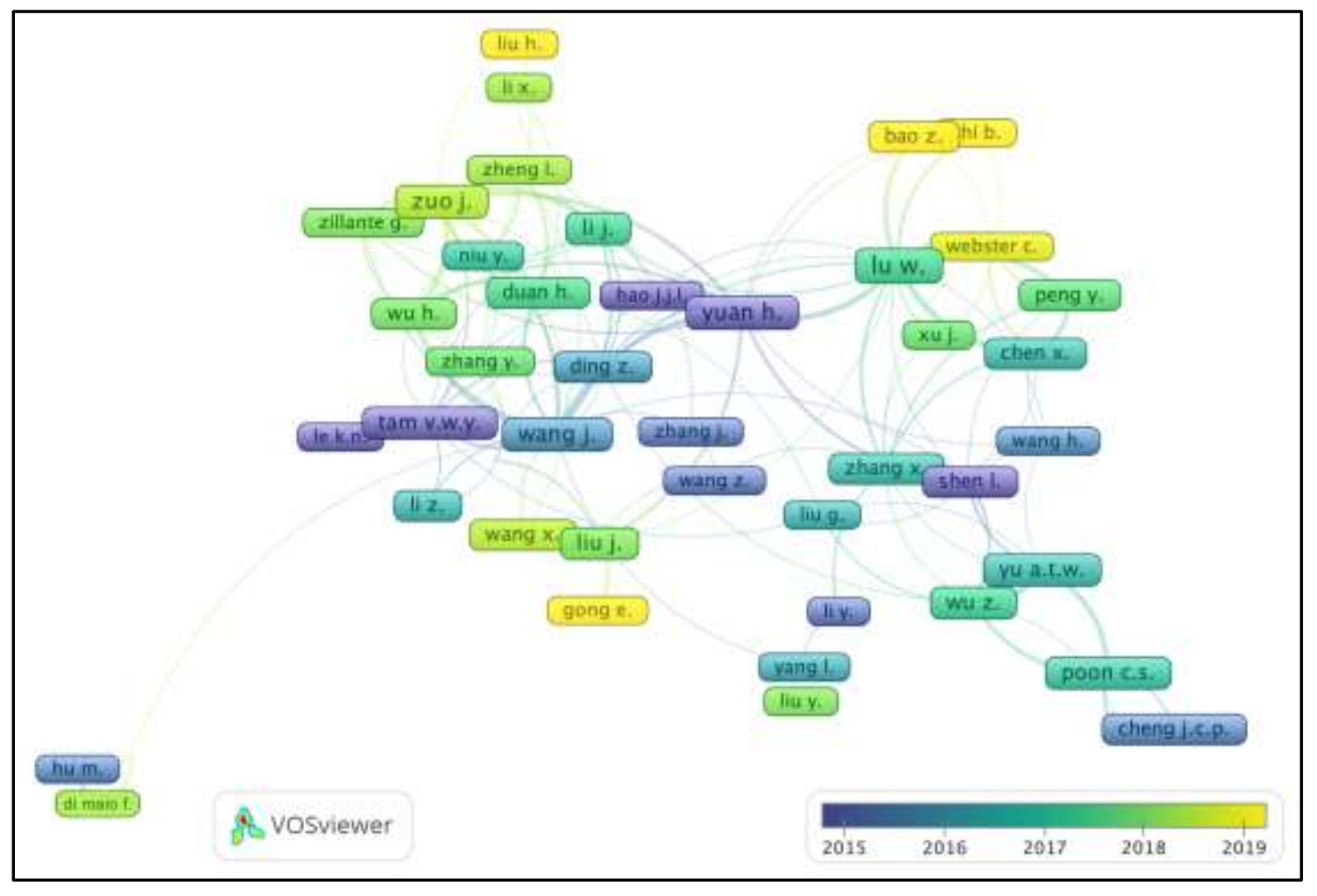

Fonte: Scopus; Vosviewer (2020) elaborado pelas Autoras (2020).

Dentre os estudos mais antigos se destacam "Critical success factors for on-site sorting of construction waste: A China study" (Wang, et al., 2010), e "Quantifying construction and demolition waste: An analytical review" (Wu, et al., 2014). O

\footnotetext{
${ }^{7} \mathrm{O}$ gradiente de cores identifica a escala temporal, sendo que as cores mais claras representam as tendências mais recentes.
} 
primeiro se destacou por explorar um campo ainda incipiente, o de fatores que resultam em uma triagem eficaz de resíduos da construção civil. Enquanto o segundo é uma revisão analítica dos métodos de quantificação de resíduos de construção e demolição.

Dentre os estudos mais recentes se destacam "Procurement innovation for a circular economy of construction and demolition waste: Lessons learnt from Sushou, China" (Bao, et al., 2019) e "Evaluation the effects of green building on construction waste management: A comparative study of three green building rating systems" (Lu, et al., 2019). Os dois contemplam termos de pesquisa mais atuais, como economia circular e edificações verdes. O que indica uma tendência de aprofundamento do assunto em um país que investe em pesquisa e desenvolvimento, buscando sanar os problemas oriundos dos RCC.

\section{Considerações Finais}

Este trabalho teve como objetivo identificar as tendências de pesquisa e apresentar a análise da evolução das publicações, por meio do mapeamento dos principais países, autores e coocorrência das principais palavras-chave. Para isto, a temática "Gestão de Resíduos da Construção Civil" foi analisada a partir da pesquisa bibliométrica, considerando o período de 2010 a 2020, por meio da base de dados Scopus. A pesquisa revelou que esta temática passou a ter mais interesse a partir de 2016 com importante destaque para o aumento contínuo das publicações nos anos seguintes, participação de 74 países, sendo a China o país com o maior volume e diversos pesquisadores considerados profícuos.

Foram identificados também os principais periódicos associados às pesquisas sobre GRCC: Waste Management, Journal of Cleaner Production e Resources Conservation and Recycling.

Com base na análise dos mapas do Vosviewer, os clusters mostraram que a rede de coautoria é liderada pela China, que possui também a obra com o maior volume de citações. A rede de coocorrência das palavras-chave mostrou que temas como generation, demolition, estimation, quantification, waste management, sustainability, environmental impact, recycling, foram consolidados por representarem conceitos básicos da GRCC e outros como reuse, reverse logistics, landfill, demolition waste, waste reduction avançaram, ampliando a sua aplicação, o que pode indicar a tendência de novas linhas de pesquisa em temas focados na estrátegia e prática de gestão inovadoras como "circular economy", "waste generation rate" e "waste recycling".

Conclui-se que os resultados desta pesquisa contribuem oferecendo uma visão abrangente sobre os caminhos da temática GRCC, para que os futuros pesquisadores consigam estruturar suas pesquisas partindo da temática mais atual e evoluir considerando uma projeção futura.

A limitação da pesquisa está relacionada ao fato de ter sido utilizado como base de dados somente a Scopus. Nesse sentido, sugere-se para as pesquisas futuras uma leitura aprofundada dos artigos levantados, como análise de conteúdo ou revisão sistemática. Acrescenta-se ainda a utilização de outras bases, preferencialmente as nacionais, a fim de compreender o cenário de pesquisa brasileiro em torno desta temática.

\section{Referências}

Abrelpe, Associação Brasileira de Empresas de Limpeza Pública e Resíduos Especiais (2019). Panorama dos Resíduos Sólidos no Brasil 2018/2019. https://abrelpe.org.br/download-panorama-2018-2019/

Ann, T. W., Poon, C. S., Wong, A., Yip, R., \& Jaillon, L. (2013). Impact of Construction Waste Disposal Charging Scheme on work practices at construction sites in Hong Kong. Waste management, 33(1), 138-146 10.1016/j.wasman.2012.09.023

Barbudo, A. et al. (2020). Recommendations for the management of construction and demolition waste in treatment plants. Environmental Science and Pollution Research, v. 27, n. 1, p. 125-132. 10.1007/s11356-019-05578-0

Bao, Z., Lu, W., Chi, B., Yuan, H., \& Hao, J. (2019). Procurement innovation for a circular economy of construction and demolition waste: Lessons learnt from Suzhou, China. Waste Management, 99, 12-21. 10.1016/j.wasman.2019.08.031 
Bovea, M. D., \& Powell, J. C. (2016). Developments in life cycle assessment applied to evaluate the environmental performance of construction and demolition wastes. Waste Management, 50, 151-172. 10.1016/j.wasman.2016.01.036

Bocken, N. M., Olivetti, E. A., Cullen, J. M., Potting, J., \& Lifset, R. (2017). Taking the circularity to the next level: a special issue on the circular economy. Journal of Industrial Ecology, 21 (3), 476 - 482. 10.1111/jiec.12606

Brasileiro, L. L., \& Matos, J. M. E. (2015). Revisão bibliográfica: reutilização de resíduos da construção e demolição na indústria da construção civil. Cerâmica, 61, 178-189. 10.1590/0366-69132015613581860

Cheng, J. C., \& Ma, L. Y. (2013). A BIM-based system for demolition and renovation waste estimation and planning. Waste management, 33 (6), $1539-1551$. 10.1016/j.wasman.2013.01.001

Chen, J., Su, Y., Si, H., \& Chen, J. (2018). Managerial areas of construction and demolition waste: a scientometric review. International journal of environmental research and public health, 15 (11), 2350. 10.3390/ijerph15112350

Córdoba, R. E. (2014). Estudo do potencial de contaminação de lixiviados gerados em aterros de resíduos da construção civil por meio de simulações em colunas de lixiviação (Doctoral dissertation, Universidade de São Paulo) https://www.teses.usp.br/teses/disponiveis/18/18138/tde-26052015-162328/en.php

Da Silva, E. L., \& Menezes, E. M. (2005). Metodologia da pesquisa e elaboração de dissertação. UFSC, Florianópolis, 4a. edição, 123 http://www.portaldeconhecimentos.org.br/index.php/por/Conteudo/Metodologia-da-pesquisa-e-elaboracao-de-dissertacao.

Ghafourian, K., Mohamed, Z., Ismail, S., Malakute, R., \& Abolghasemi, M. (2016). Current status of the research on construction and demolition waste management. Indian Journal of Science and Technology, 9 (35), 1-9. 10.17485/ijst/2016/v9i35/96231

Ghaffar, SH, Burman, M., \& Braimah, N. (2020). Caminhos para a construção circular: uma gestão integrada de resíduos de construção e demolição para recuperação de recursos. Journal of Cleaner Production, 244 , 118,710. 10.1016/j.jclepro.2019.118710

Hossain, M. U., Wu, Z., \& Poon, C. S. (2017). Comparative environmental evaluation of construction waste management through different waste sorting systems in Hong Kong. Waste Management, 69, 325-335. 10.1016/j.wasman.2017.07.043

Hou, Q., Mao, G., Zhao, L., Du, H., \& Zuo, J. (2015). Mapping the scientific research on life cycle assessment: a bibliometric analysis. The International Journal of Life Cycle Assessment, 20(4), 541-555. 10.1007/s11367-015-0846-2

Huang, K. G. (2010). China's innovation landscape. Science, 329(5992), 632-633. 10.1126 / science.1190212

Lacy, P. \& Rutqvist, J. (2015). Waste to wealth: The circular economy advantage. London, England: Palgrave MacMillan.

Laurent, A., Bakas, I., Clavreul, J., Bernstad, A., Niero, M., Gentil, E., ... \& Christensen, T. H. (2014). Review of LCA studies of solid waste management systems-Part I: Lessons learned and perspectives. Waste management, 34(3), 573-588. 10.1016/j.wasman.2013.10.045

Llatas, C. (2011). Um modelo de quantificação de resíduos de construção em projetos de acordo com a lista de resíduos europeia. Gestão de resíduos, 31 (6), 1261-1276. 10.1016/j.wasman.2011.01.023

Lu, W., Yuan, H., Li, J., Hao, J. J., Mi, X., \& Ding, Z. (2011). An empirical investigation of construction and demolition waste generation rates in Shenzhen city, South China. Waste management, 31(4), 680-687. 10.1016/j.wasman.2010.12.004

Lu, W., Webster, C., Chen, K., Zhang, X., \& Chen, X. (2017). Computational Building Information Modelling for construction waste management: Moving from rhetoric to reality. Renewable and Sustainable Energy Reviews, 68, 587-595. 10.1016/j.rser.2016.10.029

Lu, W., Chi, B., Bao, Z., \& Zetkulic, A. (2019). Evaluating the effects of green building on construction waste management: A comparative study of three green building rating systems. Building and Environment, 155, 247-256. 10.1016/j.buildenv.2019.03.050

Marques, H. F., Ribeiro, C. C., de Oliveira, D. M., Bamberg, P., \& Almeida, M. L. B. (2020). Reaproveitamento de resíduos da construção civil: a prática de uma usina de reciclagem no estado do Paraná. Brazilian Journal of Development, 6(4), 21912-21930. 10.34117/bjdv6n4-383

Mervis, J. (2010). Trends document China’s prowess. Science Indicators 327, 407. 10.1126 / science.327.5964.407

Menegaki, M., \& Damigos, D. (2018). A review on current situation and challenges of construction and demolition waste management. Current Opinion in Green and Sustainable Chemistry, 13, 8-15. 10.1016/j.cogsc.2018.02.010

Ortiz, O., Pasqualino, J. C., \& Castells, F. (2010). Environmental performance of construction waste: Comparing three scenarios from a case study in Catalonia, Spain. Waste management, 30(4), 646-654. 10.1016/j.wasman.2009.11.013

Park, J., \& Tucker, R. (2017). Overcoming barriers to the reuse of construction waste material in Australia: a review of the literature. International Journal of Construction Management, 17(3), 228-237. 10.1080/15623599.2016.1192248

Poon, C. S., Yu, A. T., Wong, A., \& Yip, R. (2013). Quantifying the impact of construction waste charging scheme on construction waste management in Hong Kong. Journal of construction engineering and management, 139(5), 466-479. 10.1061/(ASCE)CO.1943-7862.0000693

Prodanov, C. C., \& De Freitas, E. C. (2013). Metodologia do trabalho científico: métodos e técnicas da pesquisa e do trabalho acadêmico-2 ${ }^{a}$ Edição. Novo Hamburgo, Brasil: Editora Feevale.

Solís-Guzmán, J., Marrero, M., Montes-Delgado, M. V., \& Ramírez-de-Arellano, A. (2009). A Spanish model for quantification and management of construction waste. Waste Management, 29(9), 2542-2548. 10.1016/j.wasman.2009.05.009 
Research, Society and Development, v. 10, n. 12, e67101220113, 2021

(CC BY 4.0) | ISSN 2525-3409 | DOI: http://dx.doi.org/10.33448/rsd-v10i12.120113

Van Eck, N. J., \& Waltman, L. (2014). Visualizing bibliometric networks. In Measuring scholarly impact (pp. 285-320). Springer, Cham. 10.1007/978-3-31910377-8_13

Ya'cob, A. S., Zawawi, W. A., Isa, M. H., \& Othman, I. (2013). Factors that affect sustainable construction waste management efforts at site. WIT Transactions on Ecology and the Environment, 179, 1169-1176. 10.2495/SC130992

Yuan, H., \& Shen, L. (2011). Trend of the research on construction and demolition waste management. Waste management, 31(4), 670-679. 10.1016/j.wasman.2010.10.030

Yuan, H., Chini, A. R., Lu, Y., \& Shen, L. (2012). A dynamic model for assessing the effects of management strategies on the reduction of construction and demolition waste. Waste management, 32(3), 521-531. 10.1016/j.wasman.2011.11.006

Wang, J., Yuan, H., Kang, X., \& Lu, W. (2010). Critical success factors for on-site sorting of construction waste: a China study. Resources, conservation and recycling, 54(11), 931-936. 10.1016/j.resconrec.2010.01.012.

Wang, J., Wu, H., Duan, H., Zillante, G., Zuo, J., \& Yuan, H. (2018). Combining life cycle assessment and Building Information Modelling to account for carbon emission of building demolition waste: A case study. Journal of cleaner production, 172, 3154-3166. 10.1016/j.jclepro.2017.11.087

Wu, H., Zuo, J., Zillante, G., Wang, J., \& Yuan, H. (2019). Construction and demolition waste research: a bibliometric analysis. Architectural Science Review, 62(4), 354-365. 10.1016/j.wasman.2014.05.010

Wu, Z., Ann, T. W., Shen, L., \& Liu, G. (2014). Quantifying construction and demolition waste: An analytical review. Waste management, 34(9), 1683-1692. 10.1016/j.wasman.2014.05.010

Zega, C. J., \& Di Maio, Á. A. (2011). Use of recycled fine aggregate in concretes with durable requirements. Waste management, 31(11), 2336-2340. 10.1016/j.wasman.2011.06.011

Zhou, P., \& Leydesdorff, L. (2006). O surgimento da China como nação líder na ciência. Política de pesquisa, 35 (1), 83-104. 10.1016/j.respol.2005.08.006 